\title{
Discrimination learning by retardates as a function of number of implicit response trials
}

HAROLD J, FLETCHER ${ }^{1}$

UNIVERSITY OF WISCONSIN

In each of 8 sessions 10 severely retarded males received 4 new 15-trial object discrimination problems. A problem began with either $0,3,6$, or 9 initial trials during which a prompt (light cue) merely indicated the object which would subsequently be rewarded. No overt instrumental response was permitted during these prompted trials, but conventional trial-and-error procedures were followed on all remaining trials. Discriminative performance on conventional trials was directly related to the number of previously prompted trials. Confirming and extending previous research, these results forced the assumption of an implicit response which, elicited reliably by the prompt on each prompted trial, in creased the probability of a subsequent correct overt response.

Two previous studies have demonstrated that an overt response-reward sequence is not a necessary condition for the solution of object discrimination problems by retardates. House (1964), merely "demonstrating" and verbalizing reward contingencies on Trial 1, found that performance of severely retarded Ss was considerably above chance on Trial 2 . With similar Ss, Fletcher (1965) employed a prompt (i.e. a light indicating the correct object) and found significantly better than chance performance on the first trial following 6 prompted trials during which no overt response was permitted and rewards were neither displayed nor dispensed. This observation supported the assumption that the prompt reliably elicited an implicit response sequence which, in some manner, increased the probability of a subsequent correct response.

The present research was designed to confirm and extend these initial observations by relating the effect of the presumably critical implicit event to a fundamental experimental manipulation: number of trials. Specifically tested was the hypothesis that subsequent discriminative performance would be monotonically related to the number of preceding prompted trials. Method

Ten severely retarded males (MA's 26-67 mos., median $=48.5$ ), with extensive experience in prompted discrimination learning (Fletcher, 1965; Fletcher $f^{t}$ al, 1965) and ambiguous-cue problems ${ }^{2}$, were tested daily in the apparatus shown in Fig. 1.

Multidimensional junk objects were attached to $4 \times 4$ in. bases which slid in channels and covered 2 foodwells 10 in. apart. A trial began as $E$ raised a hinged door below the one-way mirror and pushed the white acrylic plastic tray to the position shown. The $S$ then placed his fingers on the object and pushed it back to expose the foodwell. In the trays front edge were two 1 in. jewelled amber lights which served as prompts.

Although familiar with the apparatus and procedures, Ss were first required to demonstrate retention of an appropriate response in the presence of the prompt. Two identical gray blocks were presented in four 12-trial sequences during which the prompt (light) was lighted in front of the baited block, and $s$ was required to make 11 correct responses (to the prompted block) in any 12-trial sequence. For these and subsequent sessions, rewarded position was randomized, raisin rewards and noncorrection procedures were used, and verbal instructions were limited to essentially "find the raisin."

The test phase consisted of 8 sessions. Each session began with a 4 -trial practice series during which the prompt was lighted in front of the baited one of the two gray blocks and instrumental responses to the prompted block were rewarded. Four 15-trial object discrimination problems followed and differed with respect to the number of initial "prompt only" trials. One problem per session had no prompting, hence conventional trial-and-error procedures on all 15 trials. A second problem began with 3 prompted trials and continued with 12 conventional trials. For a third problem 6 prompted trials were followed by 9 conventional trials. The fourth problem had 9 prompted trials and 6 conventional trials.

On all of these prompted trials, the prompt was lighted in front of the "correct" object, the tray was pushed forward, S was told to "look but don't touch" (all Ss obeyed instructions), and the tray was withdrawn

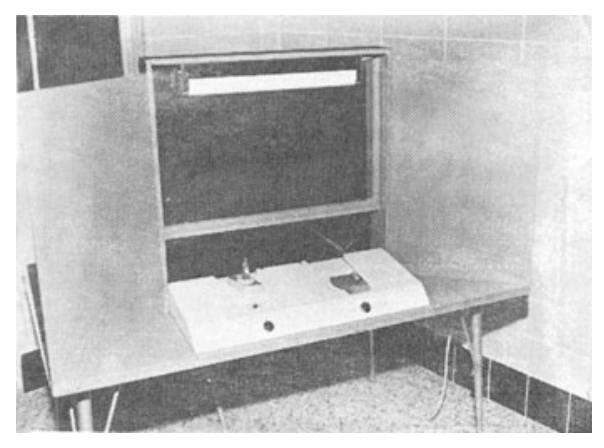

Fig. 1. Test Apparatus. 
following a normal trial's duration. Conventional trials (no prompting) always followed with interruption, and responses to "correct" object were rewarded. The order of the 4 problems was randomly determined for each $\mathrm{S}$ independently for each session.

\section{Resulis}

The most direct, hence critical, measure of the effect of previous prompted trials was the number of correct responses (over all 8 problems) on the first trial in which an overt (non-prompted) response was permitted. These first-trial data (trials 1,4 , 7 , and 10 following $0,3,6$, and 9 prompted trials, respectively) are presented in Fig. 2 .

The chance null hypothesis was first tested with each set of first trial data separately. Only apparently high, the mean of $60 \%$ on actual first trials was well within the acceptable range of sampling error $(t=1.394$; $\mathrm{df}=9 ; \mathrm{p}=.18$ ). Similar tests on the first-trial means following 3,6 , and 9 prompted trials yielded $t$ values of $3.145,6.469$, and 3.473 , respectively; each with $\mathrm{df}=9$ was significant well beyond the .01 level.

The hypothesis that performance would be monotonically related to the number of prompted trials was not supported by the first-trial performance following 9 prompted trials (Fig. 2). This one data point is somewhat suspect, however, because Ss often squirmed, looked away, or seemed annoyed during the last few of the 9 prompted trials. The protracted prevention of a response may therefore have had a relatively adverse effect on the first test trial.

An indirect, but perhaps more reliable, test of the hypothesis was available in the subsequent intraproblem performance following prompted trials. Because 9 prompted trials set a maximum limit of 6 conventional test trials common to all 4 problems, intraproblem performance scores were obtained from the first 6 conventional trials following prompted trials. Indicating a slight but virtually linear relationship, these data (Fig. 2) clearly support the expected monotonic increase in subsequent discriminative performance as a function of the number of previously prompted triais. Page's Test ${ }^{3}(\mathrm{~L}=273.5 ; \mathrm{p}<.01)$ confirmed the evident transitive relationship.

\section{Discussion}

These data demonstrate quite conclusively that an explicit response-reward sequence is not a necessary event for the solution of discrimination problems by even severely retarded humans. Although they were prevented from emitting instrumental responses and experiencing external rewards on prompted trials, these Ss did, when allowed, choose the correct object. Clearly, then, they were "responding" quite adequately to the situation in which a cue merely indicated the correct object. It seems reasonable to assume, there-

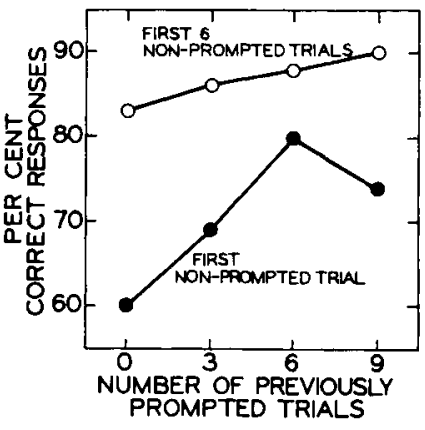

Fig. 2. Discriminative performance following prompted trials during which no instrumental response was permitted.

fore, that on each prompted trial the prompt elicited reliably and accurately an implicit response sequence which involved the prompted (or correct) object and which incrementally increased the probability of a subsequent correct discriminative response. Furthermore, the behavioral consequence of the assumed implicit response was directly related to the number of occurrences as is the probability of a correct discriminative response following conventional overt response-reward trials.

Discrimination learning theory, with its empirical foundation derived almost exclusively from conventional trial-and-error research methodology, necessarily assumes the critical event to be the effect of a reward (or nonreward) following an emitted overt instrumental response. Such a theory, in the writer's opinion, must at least struggle to account for the present results which derive from a situation manifestly involving neither an instrumental response nor a reward.

\section{References}

Fletcher, H. J. Implicit responses in discrimination learning of retardates. Psychon. Sci., 1965, 2, 229-230.

Fletcher, H. J., Davis, J. K., Or, B. M., \& Ross, P. Prompted versus trial-and-error initial obiect discrimination learning by monkeys, retardates, and preschool schildren. Psychon.Sci., $1965,2,59-60$.

House, B. J. Discrimination learning without overt response or reward in retardates. Amer. J. ment. Defic., 1964, 68, 734-740.

Page, E. B. Ordered hypotheses for multiple treatments: a significance test for linear ranks. J. Amer. Statist. Assoc., 1963, 58, 216-230.

\section{Notes}

1. The author is indebted to Harvey A. Stevens and Ronald Sindberg of the Central Wisconsin Colony administration for their generous cooperation in this research and to Mrs. Mary McNamee for her assistance.

2. Prepublication copies of this research are available upon request.

3. This test is a particularly useful and powerful alternative to the omnibus F-test when the order of treatment effects is clearly indicated in the hypothesis. Furthermore, the test requires only ordinal level of measurement. See Page (1963). 